\title{
On the limits of improved fish finding capacity and its contribution to resource conservation
}

\author{
Arne Eide \\ Norwegian College of Fishery Science \\ University of Tromsø, Norway \\ Email: arne.eide@uit.no
}

\begin{abstract}
Increased knowledge on the spatial distribution of targeted species makes it possible for the fishers to actively approaching fish dense areas, targeting fish stock characterised by non-uniform distribution. Improved fish finding technology and biological knowledge about fish behaviour and migration pattern, contribute in enhancing clever fishing. This paper investigates the limits of smart fishing by presenting a harvest model which includes a smartness parameter, representing the ability to identify the most fish dense areas of the total fish distribution area. The harvest model interacts with a fish population model where growth and distribution is represented by simple cellular automata rules. The finding indicates that smart fishing may contribute to improve stock conservation when the smartness parameter becomes sufficiently high, while a uniform distribution of fishing effort may cause stock collapse and depletion at relatively low fishing efforts. Efficient fish finding techniques could therefore be a possible tool in fish conservation, reducing the fishing mortality in the less fish dense areas, rather than a threat to the resource, since the fishing activity always will be constrained by the economic condition wherein it takes place. A 2D cellular automata model for the spatial distribution and growth of a fish population is presented. An open access fishery, targeting the species, is assumed to take place in the area and all harvest is landed in a single port within the defined area. The fishery is only restricted by biological and economic constraints made up by the population dynamics, prices and cost of fishing. The latter also includes an increasing cost due to increasing distance between the fishing area and the port. The cellular automata model of biological growth and distribution presented in this paper is inspired of some features of the Northeast Arctic Cod stock fishery, but the modelling exercise does not claim to be a reflect this fishery, rather presenting a possible implementation of cellular automata techniques in seasonal fisheries on migrating stocks. Numerical examples are provided to indicate how fishers behaviour and fish finding ability affect economic results and biological processes in open access fisheries.
\end{abstract}

Keywords: $\quad$ Bioeconomics, Spatial distribution, Open access fishery, Cellular automata modelling 


\section{INTRODUCTION}

It is well known that random fishing on a fish stock with a non-uniform distribution, will in the long run produce less catch than will effective targeting of fish dense areas, utilising fish finding equipment and other available knowledge about the distribution of the stock. However, the benefits of investing time and money in developing fishing strategies and increased knowledge on the spatial distribution of fish, obviously have some limits, as the marginal cost at some point will exceed the marginal gain of the investment. It may however not be equally obvious that the marginal benefit actually may turn negative and increasingly so, with increasing fish finding ability. This paper presents a plausible theoretical study where this actually is the case.

Some previous studies on the relationship between fish density and distribution versus distribution of fishing effort have focused on this issue from a stock assessment perspective. An early contribution was Swain and Sinclair's (1994) models for fishing effort distribution, categorising three types of effort distribution: uniform, proportional to biomass distribution and effort placed into the most fish dense area.

Swain and Wade (2002) find in a case study of a snow crab fishery that effort distribution is a rather reliable stock density indicator in this fishery, though fishing effort tended to be higher than predicted at fishing grounds near home port, and less than predicted at far distance sites.

Several studies introduce effort distribution parameters (often referred to as density, dispersion or knowledge parameters) aiming to develop statistical models to improve estimates of stock density distribution from survey data (Lewy and Kristensen, 2009), investigate the weaknesses of catch per unit of effort (CPUE) as a stock density indicator (Salthaug and Aarnes, 2003; Swain and Wade, 2003) or to focus the effect that fisher knowledge may have on catchability and stock size (Ellis and Wang, 2006). To my knowledge this paper presents the first bioeconomic study including effort distribution parameters has been published up to now.

The finding by Swain and Wade (2002) referred to above, indicates that fish density distribution alone does not explain the distribution of fishing effort even when full knowledge of fish distribution is available. Moreover, the harvest provides income, but the cost associated with harvest not only varies with fish density, but also - and increasingly so - with distance between fishing ground and home port. The model to be presented in this paper therefore addresses both of these elements, income and cost of fishing. This is achieved by assuming that the knowledge parameter is associated with the distribution of potential net revenue per unit of effort, rather than with fish density.

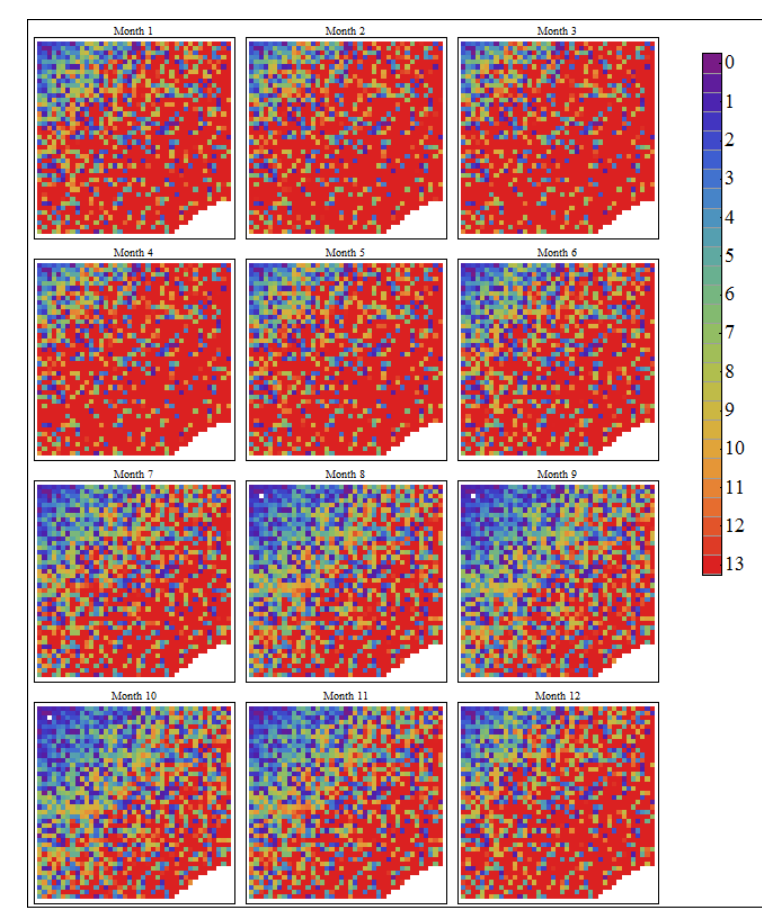

Figure 1. Assumed monthly capacity levels in the lattice. Land area is represented as white cells. January - December are represented by month number 1 - 12 respectively.

\section{MODEL}

\subsection{Seasonal carrying capacities}

The cellular automata model of biological growth and distribution of the fish species used here includes a $41 \mathrm{x} 41$ lattice where each cell is assigned a month specific growth capacity. The values are randomly chosen, assuming reduced capacity as the distance from coastal areas increases (Figure 1). The seasonal pattern is based on the seasonal variations in catchability of the Northeast Arctic Cod stock (Eide et al., 2003). The example given, though inspired
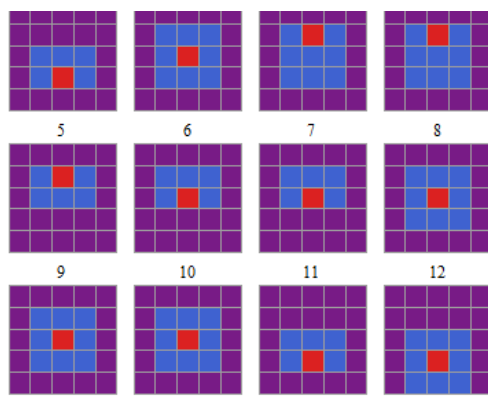

Figure 2. Rules for monthly distribution of the total biomass placed in the center cell. The values are 0.1 (magenta), 0.5 (blue) and 5.0 (red). 
by a real fishery, first of all represents a theoretic study of a modelling technique. A possible grid resolution for the Northeast Arctic Cod fishery may however be in the range of $50 \mathrm{~km}$ or less, depending on the range (diffusion) setting and the time frame (step size). The theoretical range of distance covered by a fish initially placed in the middle of a cell, then is about $125 \mathrm{~km}$ in any direction during a period of one month.

\subsection{Diffusion rules}

The cellular automata rules are based on Moore neighbourhood of range 2. Each month has a specific distribution rule, capturing seasonal migrations and diffusion to neighbouring cells. The monthly distribution rules are shown in Figure 2.

\subsection{Biomass growth}

The biomass growth within each cell is a simple linear growth model with a biomass limit determined by the capacity lattice of the given month (Figure 1). When the total biomass (current biomass plus biomass growth) after applying the distributional rule, exceeds the limit, only the fractional percentage part of the biomass is kept in the cell.

\subsection{Harvest function}

The stock-output elasticity in harvest production models is usually assumed to equal one. Most studies on stock-effort-harvest relationships indicate however that this relationship is seldom linear. Empirical studies suggest that the stock-output elasticities are more likely to be close to $1 / 2$ (Hannesson, 1983; Eide et al., 2003). In this study harvest within one cell $(h)$ is assumed to be a function of fishing effort $(e)$ and fish biomass $(x)$ within the same cell, expressed by

$h(e, x)=q e \sqrt{x}$

when $q$ is a constant catchability coefficient.

\subsection{Net revenue}

A constant unit price of harvest $(p)$ is assumed and revenue within one cell is given by $r$ :

$r(e, x)=p h(e, x)$

while the total cost of fishing within one cell $(c)$ equals

$c(e, d)=\left(c_{e}+c_{d} d\right) e$

where $c_{e}$ is a constant unit cost of effort and $c_{d}$ is a constant unit effort cost of distance from port. $d$ is a measure of the distance between fishing area (cell) and port. Here $d$ is measured as the Euclidian distance from one port to all cells as shown in Figure 3. Equation (3) is assumed to include the opportunity cost of all factors employed in harvest production.

Net revenue within one cell is hence given by

$\pi(e, x, d)=r(e, x)-c(e, d)$

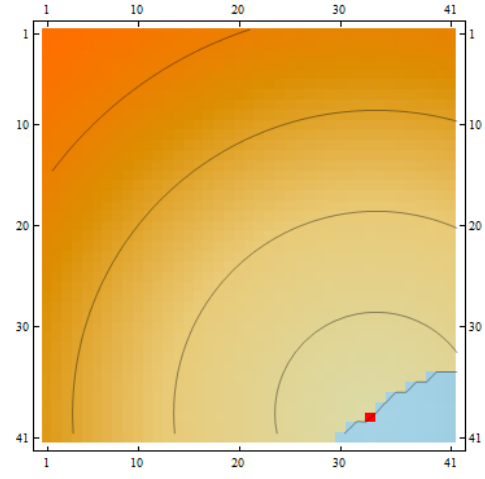

Figure 3. Distance from port (cell marked red, with coordinate $\{33,39\}$ ) increasing by colour saturation. Land area is indicated by light blue colour.

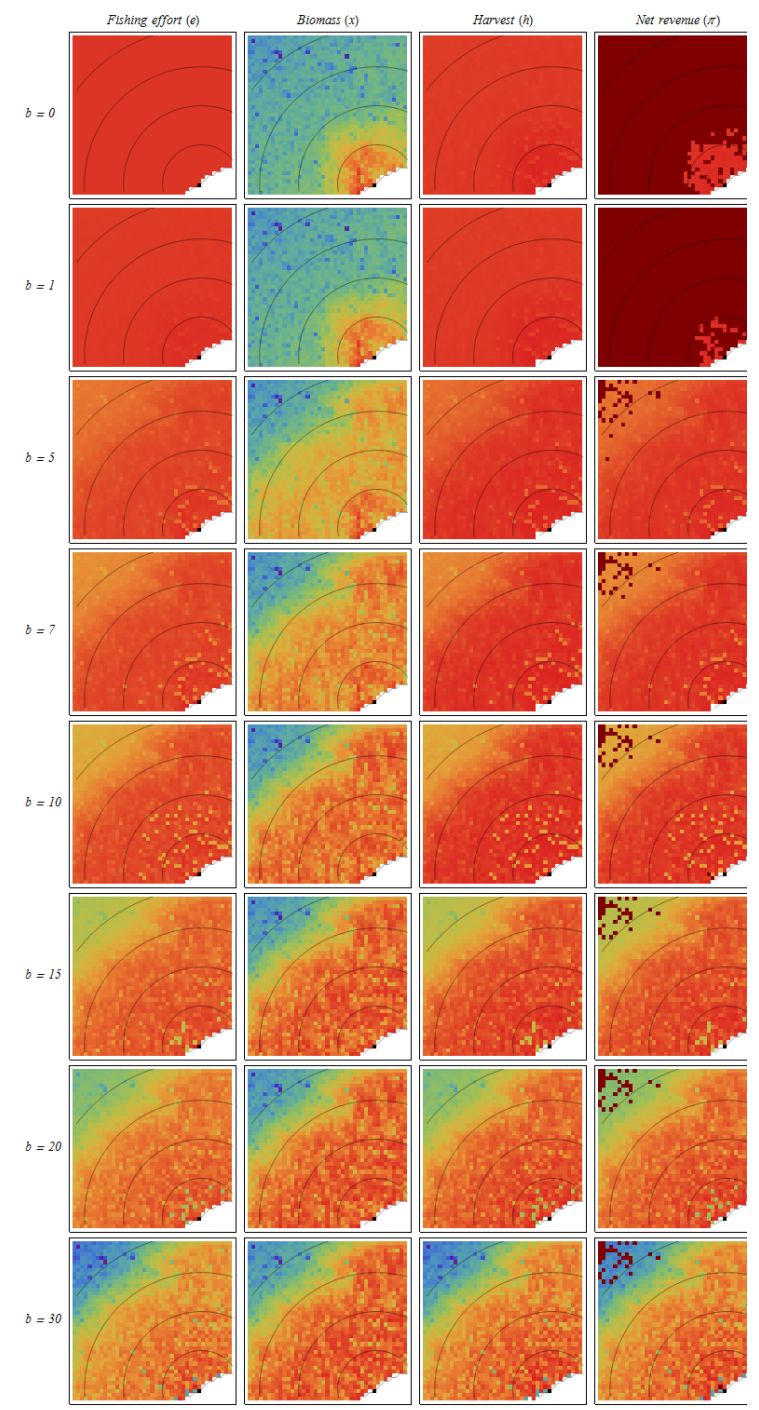

Figure 4. Distribution patterns of fishing effort, stock biomass, harvest and net revenue by different values of $b$ in December (Month 12) year 10 (see

Figure 9) of a simulation with random initial distribution of biomass and capacity levels. The legend in Figure 1 indicates the relative values of the coloured areas. 


\subsection{Smart fishing}

As the fish biomasses and distances to the cell vary, revenues and costs of fishing in different areas may differ significantly. The fishers are assumed to target the cells of the highest net revenues, but while the cost of distances are equally calculated there are limitations to their knowledge about biomass distribution. Let $E_{t}$ indicate the total effort at time $t$, being the sum of fishing effort in all $(n$, in this study $n=1681)$ cells.

$$
E_{t}=\sum_{i=1}^{n} e_{i, t}
$$

Assume the effort to be distributed in the lattice according to the ratio between revenue (2) and cost (3) within each cell, while including a distribution parameter $b$.

$$
e_{j, t}=\frac{\left(\frac{r_{j, t}}{c_{j, t}}\right)^{b}}{\sum_{i=1}^{n}\left(\frac{r_{i, t}}{c_{i, t}}\right)^{b}} E_{t}
$$

The distribution parameter $b$ could be interpreted as a smartness parameter, with increasing ability of identifying the most profitable cell by increasing $b$ value. $b=0$ gives a uniform distribution of effort

$$
e_{j, t}=\frac{\left(\frac{r_{j, t}}{c_{j, t}}\right)^{0}}{\sum_{i=1}^{n}\left(\frac{r_{i, t}}{c_{i, t}}\right)^{0}} E_{t}=\frac{E_{t}}{n}
$$

while all effort will be placed into the cell with the highest net revenue when $b$ approaches infinity. The distribution of effort at $b=1$ exactly mirrors the

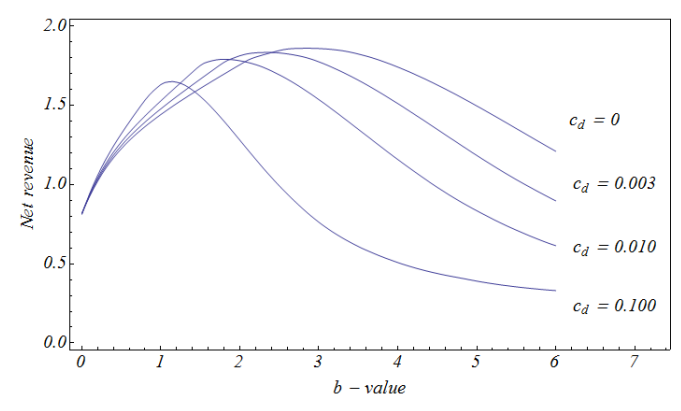

Figure 5. Net revenue obtained with different effort distributions (equation 6) and varying

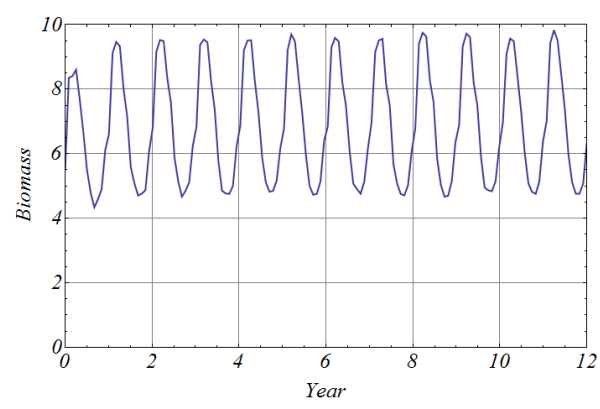

Figure 6. Biomass development of the cellular automata model over a period of 12 years without fishing.

distribution of the revenue/cost ratio. The columns in Figure 4 shows respectively the distribution of effort, biomass, harvest and net revenue (of a given example) for different values of $b$. The relative differences is reflected by colour differences.

\subsection{Unit of time}

The distribution rule (6) controlled by $b$ takes into consideration the biomass distribution through $r$ and the cost of long distances through $c$. There are however no restrictions on how units of effort may move between units of time, since these units of effort could not be traced. In the example presented here time unit is set to one month, as seen from figures 1 and 2. Effort is redistributed each time step according to rule (6).

\subsection{Open access dynamics}

Open access dynamics is assumed, with increasing or decreasing effort between time steps, depending of the economic performance of the fleet. The effort is assumed to have a fixed entry and exit rate. In the simulations presented here the monthly percentage increase (when total net revenue is positive) is assumed to equal the monthly percentage decrease (with negative net revenue) of $2 \%$.

\section{RESULTS}

\subsection{Static solutions}

For any given, constant, non-uniform area distribution of biomass and with a positive cost related to distance $\left(c_{d}\right)$, there will be a smartness parameter $(b)$ value maximising global net revenue maximum. The relation between cost related to distance and smartness is illustrated in Figure 5. The value of $b$ maximising 
net revenue decreases by increasing cost of distance. In all cases the net revenues are monotonously declining at values of $b$ beyond the maximum net revenue.

\subsection{Dynamic solutions}

As previous fishing activities affect future growth and available stock biomass, the static example provided above is not sufficient to investigate the full effect of changing the value of $b$.

The example provided includes a rather pronounced seasonal pattern, easily seen from the simulation result presented in Figure 6. This simulation is done without fishing, illustrating the natural stock condition or stock availability.

The annual fluctuations lead to a stock biomass which varies between about 5 and 9, with a mean value around 7 (biomass unit is not determined).

In order to investigate system performance under open access while varying the knowledge (smartness) parameter $(b)$, simulation-runs using the same initial conditions, were performed for different values of $b$ between 0 and 20. The main results of these simulations are shown in Figures 4, 7, 8 and 9.

Figure 4 shows distribution patterns at a point in time late in the simulation period, assuming this to give a representative snapshot of the difference in patterns between the selected $b$ values.
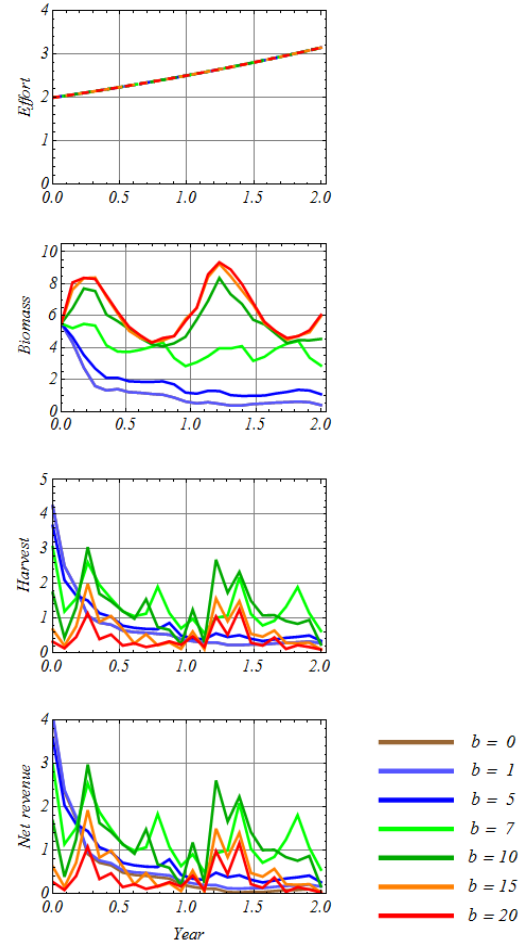

Figure 7. Two simulated years of open access fishery of different values of $b$. The full simulation time is shown in Figure 9.
Figure 7 shows a minor section (the first two years) of the full simulation presented in Figures 8 and 9. As seen from Figure 7 (hence also present in Figures 8 and 9), there is an initial decline in stock biomass for small values of $b$, while there is a temporary initial increase in stock size for $b$ values higher than 7. The latter is also reflected in lower harvest and net revenue values the first few months compared with cases with lower $b$ values. While the seasonal pattern in availability is difficult to identify at low $b$ values, it is very visible at higher $b$ values (from 7 and beyond).

Figure 8 and the surfaces presented in Figure 9 indicate that the simulation (which is deterministic, but with randomised initial biomass distribution) shows that biomass values increase with increasing value of $b$ beyond a certain point. At the same time annual fluctuations become more pronounced. Harvest and net revenue are highest for $b$ around 10 .

Fishing effort increases smoothly (with a monthly rate of $2 \%$ ) until open access levels are reached as net revenue (here representing a normal profit) approaches zero, whereby fishing effort fluctuates around the open access level. The time it takes until this happens is longer where revenue is higher.

Figure 10 presents ten simulation-runs, each covering a period of 30 years, and the results from the last ten years of each simulation are presented as dendrogram plots. The clustering process information on biomass, effort, harvest and net revenue distribution in the lattice, as well as the total levels. Chebyshev distance is chosen as clustering measure in the dendrogram plot, mimicking the biomass movement in the lattice.

\section{DISCUSSION}

The example presented above shows that the ability to target fish dense areas at short distance from home port (represented by $b$ values) significantly affects not only harvest and net revenue in the fishery, but also stock development and open access effort level. It is shown how local differences in growth capacities may be utilised to increase harvest and net revenue without stock depletion. Actually, increased ability of targeting the most profitable areas may contribute to stock conservation compared to the effect that a uniform distribution of fishing effort may have on the stock, even when the fleet operations are limited by economic constraints. 
Figure 10 shows no significant difference in biomass distribution and stock size in later years in the cases of uniform distribution of effort $(b=0)$ and effort distribution proportional to distribution of net revenue $(b=$ 1). The biomass pattern is similar to the pattern shown for catch per unit of effort, though the distances increase for the latter at $b$-values beyond 3. While the distances for successive $b$ values from 0 to 10 progressively increase for biomass, the corresponding patterns for fishing effort and net revenue indicate that the values for $\mathrm{b}$ between 1 and 5 are closer to each other than for $b$ of 0 or of 10 or more. Also worth noting is the distinct clusters of biomass patterns, as well as catch per unit of effort patterns, for $b$ values below and above 10, where the $b$ value seemingly moves into a region where the natural seasonally fluctuating biomass pattern is restored (see also Figure 8).

Increasing the ability of all fishing vessels to target the most profitable fishing grounds, leads to increased spatial concentration of the fishing activities, hence reducing the fishing pressure in other areas, given that the total fishing effort is constant. Even when increasing the total fishing effort, the fishing effort locally in less profitable areas may be reduced, if the smartness parameter $b$ is increased sufficiently., even in migrating stocks the individual fish face growth constraints and mortality in their local environment. Spawning areas may be very small compared with the total distribution area of the species, and the inclusion of such features in the model may lead to very different results. However, in such cases it may not be that obvious how recruitment success link to fishing activities on spawning grounds.

The finding in this theoretical study supports the contention that increased knowledge of the spatial distribution of fish leads to increased catches and may sustain higher fishing effort in an open access fishery than in the case of uniform distribution of effort. The distribution of fishing effort according to spatial distribution of the fish is however restricted by the cost of distance between fishing ground and home port, effectively leading to a higher exposure of local fishing grounds. Richer fishing grounds further away consequently get less exposed which may cause a spill over to the more exposed areas. This also explains the higher seasonal fluctuations seen in the cases of higher $b$ values, an effect reminiscent of the potentially increased profitability of pulse fishing, described by Hannesson (1975).

As expected increased knowledge and fish finding ability of the fleet allow higher fishing effort to develop under open access and equal market conditions. From Figures 8 and 9 (upper left panel) it is however easy to see that the increasing effort by increasing $b$ values (representing increased knowledge) reaches a maximum level, in this case at $b=10$. This may arise from at least two reasons. One obvious reason is the increased concentration of effort in smaller areas, leading to reduced per unit catches when the fishing capacity grows beyond the available biomass in the area. Even without increased cost of effort (which may be a consequence of increasing conflicts) the net revenue per unit of effort then will decline.

The lower right panel in Figure 9, displaying the time-b-value surface of net revenue from the simulations, shows surprisingly high net revenues in open access for high $b$-values, in particular for the values leading to higher fishing effort. One interpretation may be that high capacity fleets (particularly for $b=10$ ) manage to take greater advantage of the stock dense periods of the annual stock fluctuations. The high peaks are however damped over years, but still remain significant after the 12 years presented in Figure 9. As seen from the upper right panel in this figure, the stock fluctuations persist, and even become more pronounced, at $b$-values above 10, but the peak values of net revenues for these situations nevertheless decline, probably also because of the significant reduction in open access levels of fishing effort at $b$-values above 10 . The

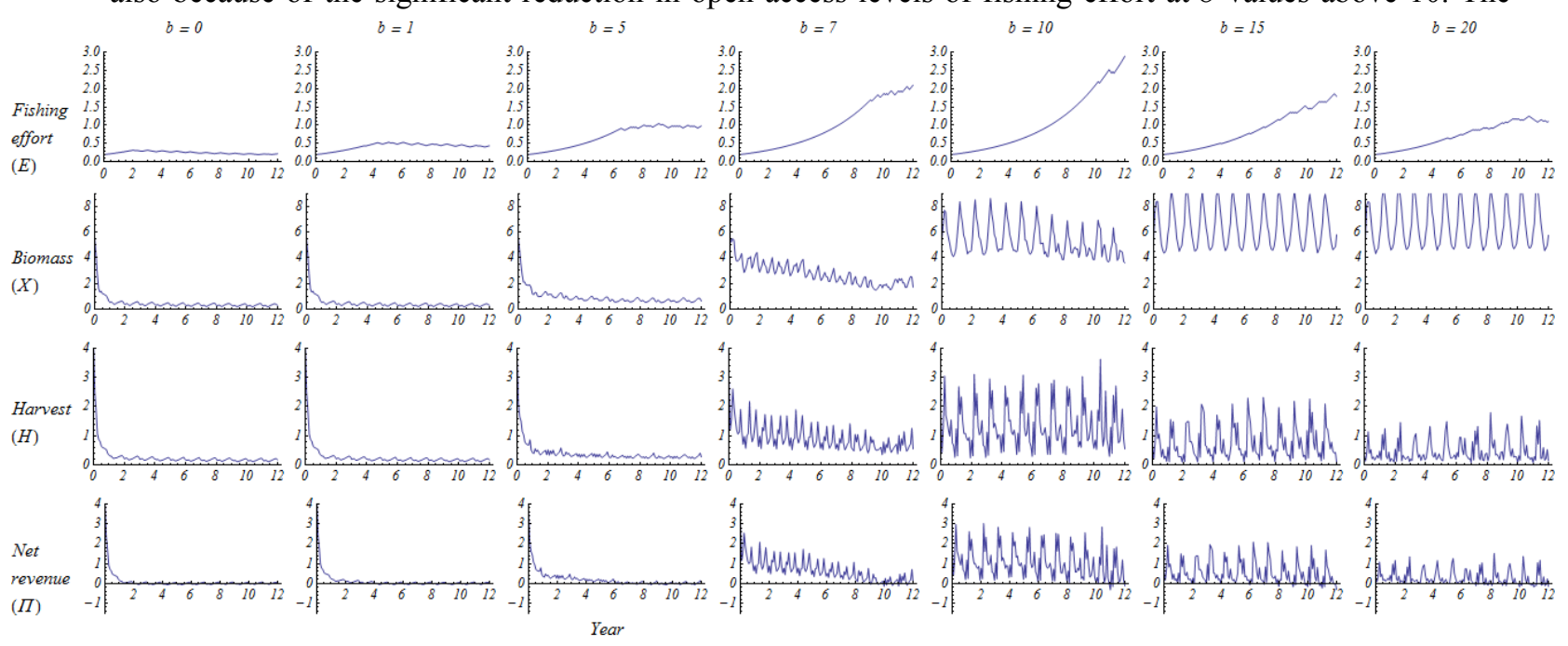

Figure 8. Distribution of fishing effort (equation 6) by varying the value of $b$. 
peak values seen in Figure 9 also is visible in the dendrograms shown in Figure 10, indicating at which $b$-value (close to 15 ) the significant change in effort, biomass, CPUE, harvest and net revenue occurs. While the pattern of effort and net revenue of $b=1$ is closer to $b=3$ and $b=5$ than $b=0$, the patterns of biomass, CPUE and harvest show almost no difference between $b=0$ and $b=1$.

The dendrograms of biomass and CPUE in Figure 10 also indicate that CPUE may be a good indicator of biomass at varying values of $\mathrm{b}$, though the relative differences of distances are not the same. This is however expected when using a non-linear catch equation (1).

This modelling example has shown that while smart fishing contributes in increased harvest and net revenue in open access fisheries, it may also effectively protect areas of low profitability (low density or long distance). This effect may increase with increasing ability of targeting the most profitable areas. At some point the increased smartness will concentrate the fleet into areas, though being fish dense, not having the capacity to serve the total effort. Hence the total effort is not efficiently used and the harvest will decline as a consequence of relative overcapacity even if the stock is in good shape. The extreme of $b=\infty$ illustrates the point, placing the whole fleet into the single cell on the
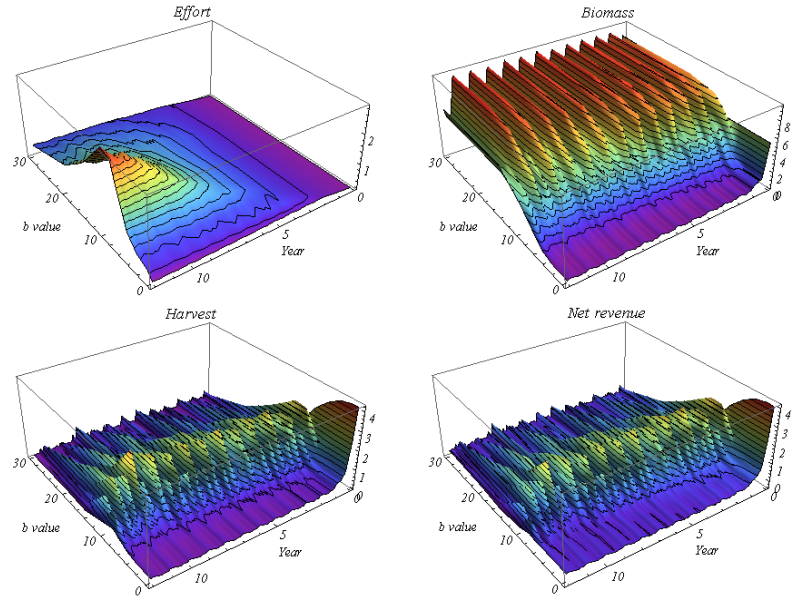

Figure 9. Simulated surfaces displaying how effort, biomass, harvest and net revenue varies with time and $b$ values. The surfaces is based on simulations with $b$ values of $0,1,3,5,7,8,10,15,20$ and 30 .

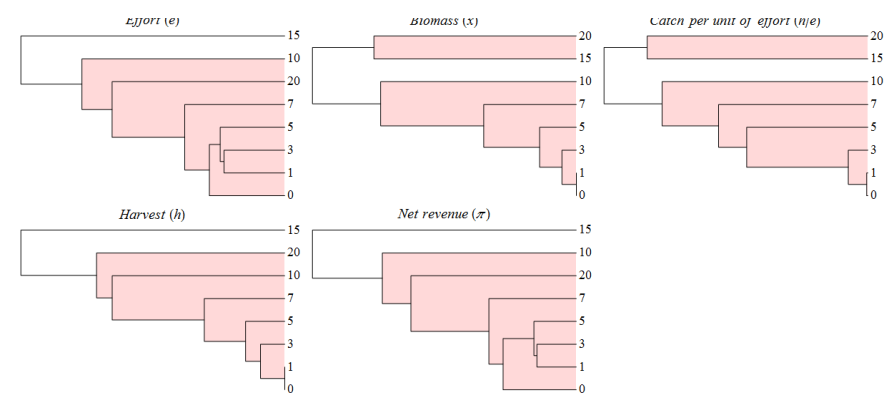

Figure 10. Chebyshev distance dendrogram plots of the last ten year of 1030 year simulations, each sharing the same parameter setting but different random initial biomass distributions. The numbers refer to the value of smartness parameter $b$. Clustering level 2 is indicated by coloured blocks. margin holding the highest cell biomass. The only this biomass is available for the fleet while the rest of the stock biomass remains unexploited.

\section{REFERENCES}

Eide, A., Skjold, F., Olsen, F. and Flaaten, O. (2003). Harvest Functions: The Norwegian Bottom Trawl Cod Fisheries, Marine Resource Economics 18: 81-93.

Ellis, N. and Y-G. Wang (2007). Effects of fish density distribution and effort distribution on catchability. ICES J. Mar. Sci. 64 (1): 178-191. doi: 10.1093/icesjms/fsl015

Hannesson, R. (1975). Fishery Dynamics: A North Atlantic Cod Fishery. The Canadian Journal of Economics 8:151 - 173 .

Lewy, P. and K. Kaspersen (2009). Modelling the distribution of fish accounting for spatial correlation and overdispersion. Canadian Journal of Fisheries and Aquatic Sciences, 66: 1809-1820.

Salthaug, A., and S. Aanes (2003). Catchability and the spatial distribution of fishing vessels. Canadian Journal of Fisheries and Aquatic Sciences, 60: 259-268.

Swain, D. P. and A. F. Sinclair (1994). Fish distribution and catchability: What is the appropriate measure of distribution? Canadian Journal of Fisheries and Aquatic Sciences, 51: 1046-1054.

Swain, D. P. and E. J. Wade (2003). Spatial distribution of catch and effort in a fishery for snow crab (Chionoecetes opilio): tests of predictions of the ideal free distribution. Canadian Journal of Fisheries and Aquatic Sciences, 60: 897-909. 